\title{
Optical properties of graphene nanoribbons: The role of many-body effects
}

\author{
Deborah Prezzi,,${ }^{1,2, *}$ Daniele Varsano, ${ }^{1}$ Alice Ruini ${ }^{1,2}$ Andrea Marini, ${ }^{3}$ and Elisa Molinari ${ }^{1,2}$ \\ ${ }^{1}$ INFM-CNR-S3, National Center on NanoStructures and BioSystems at Surfaces, I-41100 Modena, Italy \\ ${ }^{2}$ Dipartimento di Fisica, Università di Modena e Reggio Emilia, I-41100 Modena, Italy \\ ${ }^{3}$ Dipartimento di Fisica, Università di Roma "Tor Vergata," I-0133 Roma, Italy
}

(Received 6 April 2007; revised manuscript received 3 December 2007; published 7 January 2008; corrected 14 January 2008)

\begin{abstract}
We investigate from first principles the optoelectronic properties of nanometer-sized armchair graphene nanoribbons (GNRs). We show that many-body effects are essential to correctly describe both energy gaps and optical response. As a signature of the confined geometry, we observe strongly bound excitons dominating the optical spectra, with a clear family-dependent binding energy. Our results demonstrate that GNRs constitute one-dimensional nanostructures whose absorption and luminescence performance can be controlled by changing both family and edge termination.
\end{abstract}

DOI: 10.1103/PhysRevB.77.041404

PACS number(s): 73.22.-f, 78.30.Na, 78.67.-n

The recent discovery of stable, single-layer graphene ${ }^{1,2}$ has prompted investigation on a novel graphitic quasi-onedimensional (1D) nanostructure, i.e., graphene nanoribbons (GNRs). These systems have been theoretically studied in the past decade ${ }^{3-7}$ as simplified models of defective nanotubes and graphite nanofragments. However, only very recently have isolated nanometer-sized GNRs actually been synthetized by etching larger graphene samples, or by chemical vapor deposition growth on suitably patterned surfaces. ${ }^{8-10}$ The production techniques used in these pioneering works are expected to become highly controllable, opening up new avenues for both fundamental nanoscience and nanotechnology applications.

One of the most striking features of GNRs is the high sensitivity of their properties to the details of the atomic structure. ${ }^{3-5,11-14}$ In particular, the edge shape dictates their classification into armchair $(A)$, zigzag $(Z)$, or chiral $(C)$, thus determining their energy band gaps. In addition to an overall decrease of energy gap with increasing ribbon width, also observed experimentally, ${ }^{10}$ theoretical studies predict a superimposed oscillation feature, ${ }^{11-13}$ which is maximized for $A$-GNRs. According to this behavior, $A$-GNRs are further classified into three distinct families, i.e., $N=3 p-1, N=3 p$, and $N=3 p+1$, with $p$ integer, where $N$ indicates the number of dimer lines across the ribbon width. This fine sensitivity to the atomic configuration raises the opportunity to tailor the optoelectronic properties of $A$-GNRs by appropriately selecting both ribbon family and width.

In spite of this interest, previous theoretical studies of the electronic (see, e.g., Refs. 4, 13, and 14) and optical properties $^{12}$ of GNRs were based only on the independentparticle approximation or on semi-empirical calculations. However, many-body effects are expected to play a key role in low-dimensional systems ${ }^{15-18}$ due to enhanced electronelectron correlation. Motivated by this theoretical issue and by recent experimental progress pursuing the potential of GNRs for nanotechnology applications, we have carried out $a b$ initio calculations to study the effects of many-body interactions on the optical spectra of 1-nm-wide A-GNRs belonging to different families.

In this paper, we show that a sound and accurate description of the optoelectronic properties of $A$-GNRs must include many-body effects. We demonstrate that there are many signatures of the nonlocal correlations occurring in these confined systems. First of all, quasiparticle corrections are found to be strongly state dependent. Moreover, the optical response of $A$-GNRs is dominated by prominent excitonic peaks, with a complex bright-dark structure, which would not have been expected in an independent-particle framework. Both quasiparticle corrections and exciton binding energies are found to exhibit an oscillating behavior, according to the family classification. Finally, the electronic and optical properties of hydrogen-passivated $A$-GNRs are compared with those of clean-edge ribbons: including many-body effects allows us to single out the impact of this edge modification on absorption and luminescence.

The first-principles calculation of the optical excitations is carried out using a many-body perturbation theory approach, based on a three-step procedure. ${ }^{19}$ As a preliminary step, we obtain the ground-state electronic properties of the relaxed system, by performing a density-functional theory (DFT) supercell calculation, within the local density approximation (LDA). ${ }^{20,21}$ Second, the quasiparticle corrections to the LDA eigenvalues are evaluated within the $G_{0} W_{0}$ approximation for the self-energy operator, where the LDA wave functions are used as good approximations for the quasiparticle ones, and the screening is treated within the plasmon-pole approximation. $^{22}$ Third, the electron-hole interaction is included by solving the Bethe-Salpeter (BS) equation in the basis set of quasielectron and quasihole states, where the static screening in the direct term is calculated within the random-phase approximation. Only the resonant part of the BS Hamiltonian is taken into account throughout the calculations (Tamm-Dancoff approximation), since we have verified that the inclusion of the coupling part does not significantly affect the absorption spectra (i.e., the differences are below the computational accuracy). Moreover, only the case of light polarized along the ribbon axis is examined, as a significant quenching of optical absorption is known to occur in $1 \mathrm{D}$ systems for polarization perpendicular to the principal axis. ${ }^{23}$ All the $G W$ BS calculations are performed with the code SELF. ${ }^{24,25}$ To treat an isolated system in the supercell approach, we consider a separation of 40 a.u. between images in the directions perpendicular to the ribbon axis. Moreover, in both $G W$ and BS calculations, we truncate the long- 
a )

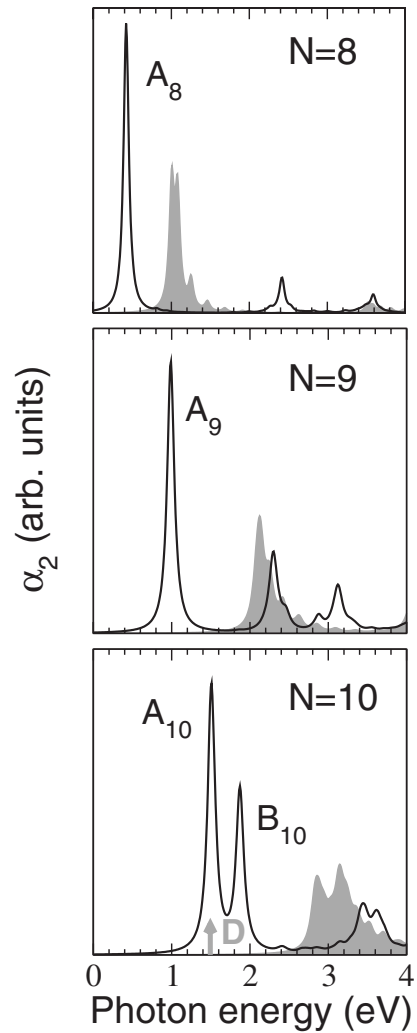

b )

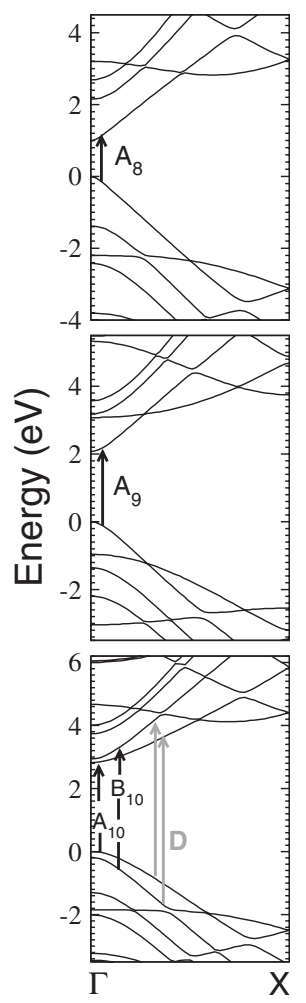

Wavevector

FIG. 1. (a) Optical absorption spectra of 1-nm-wide hydrogenpassivated GNRs: $N=8$ (1.05 nm wide), $9(1.17 \mathrm{~nm})$, and 10 $(1.29 \mathrm{~nm})$. In each panel, the solid line represents the spectrum with electron-hole interaction, while the spectrum in the single-particle picture is in gray. All the spectra are computed introducing a Lorentzian broadening. (b) Quasiparticle band structures.

range screened Coulomb interaction between periodic images, in order to avoid nonphysical interactions. ${ }^{26}$ Due to the rectangular geometry of the system, we use a box-shaped truncation. ${ }^{27}$

We start by considering 1-nm-wide hydrogen-passivated $A$-GNRs belonging to different families, namely, $N$ $=8,9,10$. Figure 1 (a) depicts their calculated optical absorption spectra, while the quasiparticle band structures are shown in Fig. 1(b). All the results are summarized in Table I. The quasiparticle $G W$ corrections open the LDA energy gaps at $\Gamma$ by $0.72,1.32$, and $1.66 \mathrm{eV}$ for $N=8,9$, and 10 , respectively. These energy corrections are larger than those of bulk semiconductors with similar LDA gaps, due to the enhanced Coulomb interaction in low-dimensional systems and the reduced screening. In addition, a family modulation of the corrections can be noticed, with larger corrections for the GNRs with larger LDA gaps. The gap opening is accompanied by an overall stretching of the banstructure of $17-22 \%$, similar to the value found for graphene $(\sim 20 \%){ }^{28}$

In the absence of electron-hole $e-h$ interaction, such a band structure would result in the optical absorption spectra depicted in gray [Fig. 1(a)], characterized by prominent 1D Van Hove singularities. The inclusion of the excitonic effects (solid black line) dramatically modifies both the peak posi-

TABLE I. Energy gap (second and third columns) and peak position (fourth column) for $N=8,9$, and $10 A$-GNRs, with (-H) and without hydrogen passivation of the edge sites. The relative binding energies are reported in the last column. All the values are in $\mathrm{eV}$.

\begin{tabular}{lllll}
\hline \hline$N$ & LDA & $G W$ & \multicolumn{1}{c}{ BS } & $E_{b}$ \\
\hline $8-\mathrm{H}$ & 0.28 & 1.00 & 0.42 & 0.58 \\
8 & 0.50 & 1.59 & 0.71 & 0.88 \\
$9-\mathrm{H}$ & 0.78 & 2.10 & 0.99 & 1.11 \\
9 & 0.56 & 1.50 & 0.64 & 0.86 \\
$10-\mathrm{H}$ & 1.16 & 2.82 & $1.51,1.87$ & $1.31,0.95$ \\
10 & 1.09 & 2.64 & $1.46,1.68$ & $1.18,0.96$ \\
\hline \hline
\end{tabular}

tion and absorption line shape, giving rise to individual excitonic states below the onset of the continuum, with binding energy of the order of the eV. The lowest-energy absorption peaks for $N=8$ and 9 , labeled $A_{8}$ and $A_{9}$, have the same character: in both cases, the principal contribution comes from optical transitions between the last valence and first conduction bands, localized in $k$ space near the $\Gamma$ point [Fig. 1(b)]. The binding energies for these lowest optically active excitons are 0.58 and $1.11 \mathrm{eV}$ for $N=8$ and 9 , respectively. As compared to the first two systems, the $N=10$ GNR shows a richer low-energy spectrum. Each noninteracting peak gives rise to a bright excitonic state [arrows $A_{10}$ and $B_{10}$ in Fig. 1(b)], with binding energies of 1.31 and $0.95 \mathrm{eV}$. In addition, the mixing of dipole-forbidden transitions between the same bands [arrows $D$ in Fig. 1(b)] is responsible for an optically inactive exciton degenerate in energy with $A_{10}$. The $D$ state thus provides a competing path for nonradiative decay of optical excitations, which could affect the luminescence yield of the system. This feature results from transitions between pairs of bands very close in energy to each other, and is therefore expected to be a common outcome for all $N=3 p+1$ GNRs. Hence, the presence of low-energy dark excitons in GNRs is strongly dependent on the family, contrary to the case of carbon nanotubes, where this is instead a common feature. ${ }^{29}$

A further insight into the effects of electron-hole interaction is provided by the evaluation of the resulting spatial correlations. In Fig. 2, we plot the in-plane probability distribution of the electron for a fixed hole position (black dot), corresponding to the lowest excitonic state in the $N=9$ case. While the electron distribution extends over the whole ribbon width, the modulation of the exciton wave function

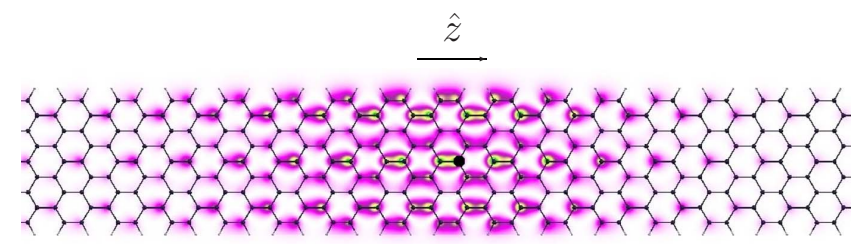

FIG. 2. (Color online) In-plane spatial distribution of the electron for a fixed hole position (black dot), corresponding to the lowest excitonic peak in the $N=9$ case. The spatial density is averaged over the direction orthogonal to the ribbon plane. Dimension of the panel: $1.2 \times 6.4 \mathrm{~nm}^{2}$. 
a)

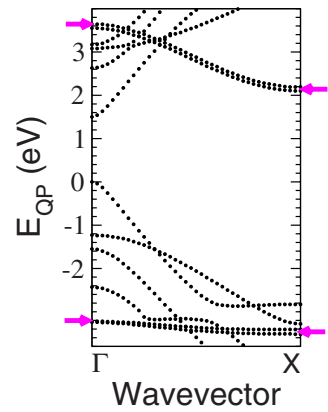

b )

C )

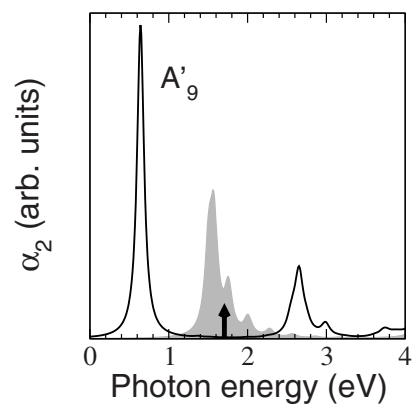

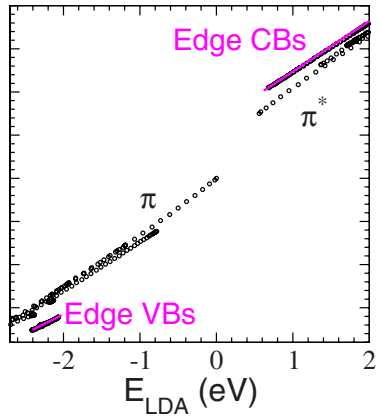

d)

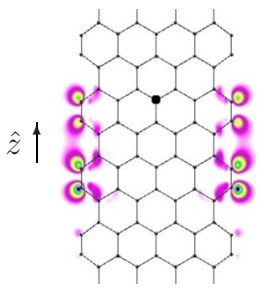

FIG. 3. (Color online) (a) Quasiparticle band structure of the $N=9$ hydrogen-free GNR. Arrows indicate the edge-related singleparticle bands. (b) Plot of $G W$ quasiparticle energies vs LDA energies for different states and $k$ points. (c) Optical absorption spectrum, with (black) and without (gray) excitonic effects. The black arrow indicates the position of the dark edge-related exciton. Its excitonic wave function is depicted in (d), whose dimension is $1.0 \times 2.2 \mathrm{~nm}^{2}$.

$\left|\psi\left(\mathbf{r}_{e} ; \mathbf{r}_{h}\right)\right|^{2}$ along the ribbon axis is entirely determined by the Coulomb interaction, confirming the truly quasi-1D character of the optical excitations. Similar wave functions (not reported here) for the lowest excitons have been obtained for GNRs of different families, with spatial extensions ${ }^{30}$ of about 34,23 , and $18 \AA$ for $N=8,9$, and 10 , respectively.

While for Z-GNRs the edge structure can be modified only by substituting hydrogen atoms with other functional groups, ${ }^{31,32}$ A-GNRs are found to be stable also if hydrogen is simply removed from the edges; in fact, this variation of the structure has often been suggested for ribbons obtained by high-temperature treatments or by dehydrogenation of hydrocarbons. ${ }^{6,12,33}$ Therefore, we now analyze the case of clean-edge nanoribbons, which will allow us to further explore the role played by edge effects in the optoelectronic properties. Our results are summarized in Fig. 3 and Table I. As expected, the hydrogen removal leads to a major edge reconstruction, with the appearence of carbynelike structures. In fact, the bond length for the edge dimers is reduced from $1.36 \AA$ for the passivated ribbons to $1.23 \AA$ for the clean ones, pointing to the formation of $\mathrm{C}-\mathrm{C}$ triple bonds at the edges. This edge modification leads to a variation of the energy gaps, such that the distinction between $N=3 p-1$ and $N=3 p$ families vanishes, in agreement with previous results. ${ }^{12}$

In Fig. 3(a), we report the quasiparticle band structure for the $N=9$ bare ribbon. The main difference with respect to its passivated counterpart is the presence of edge-related bands (see arrows) in the low-energy optical window. These states show the same energy dispersion and real-space localization, irrespective of both family and size, already in the LDA framework: ${ }^{34}$ due to this independence of bulk properties, their presence is reasonably expected for all nonpassivated ribbons. The self-energy corrections to the LDA eigenvalues are similar to those of the passivated systems for the $\pi$ and $\pi^{*}$ bulk states [Fig. 3(b), black circles], that is, the correction is linear in energy, besides a rigid gap opening. Instead, the edge states [Fig. 3(b), magenta (gray) lines] show quite a different correction, having both larger slope and larger energy shift. This behavior is to be ascribed to the different degree of real-space localization between bulk and edge states, and it can be singled out by virtue of the nonlocal character of the self-energy operator in the $G W$ framework, which is not correctly described within the LDA.

The aformentioned modification of the band structure results in a correspondent blueshift $(N=8)$ or redshift $(N=9)$ of the lowest excitonic peak, with $A_{8}^{\prime}$ and $A_{9}^{\prime}$ becoming almost degenerate, with binding energies of about $0.9 \mathrm{eV}$. For the case of $N=10$, we find an inversion of the first and second conduction bands, which results in the $B_{10}^{\prime}$ peak lying below $A_{10}^{\prime}$ and $D^{\prime}$ being almost degenerate in energy with $B_{10}^{\prime}$. In addition, the edge states introduce an optically inactive exciton, which arises from transitions among several bulk valence bands and the conduction edge states over the whole Brillouin zone. This edge exciton is present in all the studied nanoribbons and is located at about 1.4-1.7 eV [black arrow in Fig. 3(c)], with very little dependence on family and size. ${ }^{34}$ This results in the edge exciton being above the first excitonic peak for $N=8$ and 9 , and between the first and the second peaks for $N=10$. We remark that the accurate evaluation of quasiparticle corrections within the $G W$ approximation, i.e., beyond the usual approximation based on a uniform band stretching on top of a rigid energy shift, is crucial to determine the exact energy position of the dark edge excitons relative to the bright ones. To better understand the character of the edge-related dark state, we plot its excitonic wave function for the case $N=9$ in Fig. 3(d). The mixing of transitions over the whole Brillouin zone induces a strong localization of the edge exciton along the ribbon axis, with an extent of only $\sim 5 \AA$, which is $4-7$ times smaller than the Wannier-like bulk excitons (see Fig. 2).

In summary, we have found that the analysis of the electronic and optical features of GNRs requires a state-of-the-art approach within many-body perturbation theory, and beyond the DFT framework. Many-body effects reveal that nanosized $A$-GNRs retain a quasi-1D character, which induces the suppression of the Van Hove singularity, typical of noninteracting 1D systems, and the appearence of strong excitonic peaks in the optical absorption spectrum. The lowest excited states in GNRs are Wannier-like excitons and their binding energy as well as their luminescence properties are strongly dependent on the ribbon family. Moreover, many-body effects are shown to be crucial to single out nonlocal correlations and edge-related dark excitons in nonpassivated GNRs. We finally demonstrate which GNRs are intriguing systems 
with tunable optoelectronic features, which we quantitatively evaluate through our calculations. The present study calls for experiments addressing the optical response of GNRs: A combined theoretical and experimental understanding of ribbon size, family, and edge termination as control parameters for their performance can be considered as the first step toward the design of graphene-based applications in nanoscale optoelectronics.

Note added. Recently, we became aware of a related the- oretical study by L. Yang et al. ${ }^{35}$ on the $N=10,11$, and 12 $A$-GNRs, obtaining similar trends to those found in the present work for the passivated systems.

We are grateful to A. Ferretti, A. C. Ferrari, A. Grueneis, S. Piscanec, and A. Rubio for stimulating discussions. We acknoweledge CINECA CPU time granted through INFMCNR. D.V. thanks the European Nanoquanta NoE (Grant No. NMP4-CT-2004-500198).
*Corresponding author. prezzi.deborah@unimore.it

${ }^{1}$ K. S. Novoselov et al., Science 306, 666 (2004).

${ }^{2}$ For a recent review on graphene, see, e.g., A. K. Geim and K. S. Novoselov, Nat. Mater. 6, 183 (2007).

${ }^{3}$ M. Fujita, K. Wakabayashi, K. Nakada, and K. Kusakabe, J. Phys. Soc. Jpn. 65, 1920 (1996).

${ }^{4}$ K. Nakada, M. Fujita, G. Dresselhaus, and M. S. Dresselhaus, Phys. Rev. B 54, 17954 (1996).

${ }^{5}$ K. Wakabayashi, M. Fujita, H. Ajiki, and M. Sigrist, Phys. Rev. B 59, 8271 (1999).

${ }^{6}$ T. Kawai, Y. Miyamoto, O. Sugino, and Y. Koga, Phys. Rev. B 62, R16349 (2000).

${ }^{7}$ K. Kusakabe and M. Maruyama, Phys. Rev. B 67, 092406 (2003).

${ }^{8}$ Z. Chen, Y.-M. Lin, M. J. Rooks, and P. Avouris, arXiv:cond-mat/ 0701599 (unpublished).

${ }^{9}$ T. Tanaka et al., Solid State Commun. 123, 33 (2002).

${ }^{10}$ M. Y. Han, B. Ozyilmaz, Y. Zhang, and P. Kim, Phys. Rev. Lett. 98, 206805 (2007).

${ }^{11}$ M. Ezawa, Phys. Rev. B 73, 045432 (2006).

${ }^{12}$ V. Barone, O. Hod, and G. E. Scuseria, Nano Lett. 6, 2748 (2006).

${ }^{13}$ Y.-W. Son, M. L. Cohen, and S. G. Louie, Phys. Rev. Lett. 97, 216803 (2006).

${ }^{14}$ L. Pisani, J. A. Chan, B. Montanari, and N. M. Harrison, Phys. Rev. B 75, 064418 (2007).

${ }^{15}$ A. Ruini, M. J. Caldas, G. Bussi, and E. Molinari, Phys. Rev. Lett. 88, 206403 (2002).

${ }^{16}$ M. Rohlfing and S. G. Louie, Phys. Rev. Lett. 82, 1959 (1999).

${ }^{17}$ E. Chang, G. Bussi, A. Ruini, and E. Molinari, Phys. Rev. Lett. 92, 196401 (2004).

${ }^{18}$ C. D. Spataru, S. Ismail-Beigi, L. X. Benedict, and S. G. Louie, Phys. Rev. Lett. 92, 077402 (2004).

${ }^{19}$ For a recent review, see, e.g., G. Onida, L. Reining, and A. Rubio,
Rev. Mod. Phys. 74, 601 (2002).

${ }^{20}$ S. Baroni, A. Dal Corso, S. de Gironcoli, and P. Giannozzi, http:// www.pwscf.org

${ }^{21}$ The DFT LDA calculations are carried out using separable normconserving pseudopotentials and a plane-wave basis set. A kinetic energy cutoff of $50 \mathrm{Ry}$ is employed. Each atomic structure was fully relaxed, until forces acting on atoms were less than $0.01 \mathrm{eV} / \AA$.

${ }^{22}$ R. W. Godby and R. J. Needs, Phys. Rev. Lett. 62, 1169 (1989).

${ }^{23}$ A. G. Marinopoulos, L. Reining, A. Rubio, and N. Vast, Phys. Rev. Lett. 91, 046402 (2003).

${ }^{24}$ A. Marini et al., The SELF project, http://www.fisica.uniroma2.it/ $\sim$ self/

${ }^{25}$ An 80 -k-point sampling of the one-dimensional Brillouin zone is used for both $G W$ and BS calculations, which allows for converged results better than $0.1 \mathrm{eV}$.

${ }^{26}$ C. A. Rozzi, D. Varsano, A. Marini, E. K. U. Gross, and A. Rubio, Phys. Rev. B 73, 205119 (2006).

${ }^{27}$ D. Varsano and A. Marini (unpublished).

${ }^{28}$ C. Attaccalite, L. Wirtz, and A. Rubio (private communication); A. Grueneis et al. (unpublished).

${ }^{29}$ Carbon nanotubes present a different kind of dark state arising from the mixing of $K-K^{\prime}$ transitions, which are not present in GNRs due to the absence of $R_{U}$ symmetry.

${ }^{30} \mathrm{We}$ calculate the extension as the full width at half maximum of the probability distribution.

${ }^{31}$ D. Gunlycke, J. Li, J. W. Mintmire, and C. T. White, Appl. Phys. Lett. 91, 112108 (2007).

${ }^{32}$ O. Hod, V. Barone, J. E. Peralta, and G. E. Scuseria, Nano Lett. 7, 2295 (2007).

${ }^{33}$ L. R. Radovic and B. Bockrath, J. Am. Chem. Soc. 127, 5917 (2005).

${ }^{34} \mathrm{We}$ checked the results for a few other cases up to $2 \mathrm{~nm}$.

${ }^{35}$ L. Yang, M. Cohen, and S. Louie, Nano Lett. 7, 3112 (2007). 